\title{
Laser-written optical-path redirected waveguide device for optical back-plane interconnects
}

\section{AUTHOR(S):}

Hiramatsu, S; Mikawa, T; Ibaragi, O; Miura, K; Hirao, $\mathrm{K}$

\section{CITATION:}

Hiramatsu, S ... [et al]. Laser-written optical-path redirected waveguide device for optical back-plane interconnects. IEEE PHOTONICS TECHNOLOGY LETTERS 2004, 16(9): 20752077

\section{ISSUE DATE:}

2004-09

URL:

http://hdl.handle.net/2433/39937

\section{RIGHT:}

(c)2004 IEEE. Personal use of this material is permitted. However, permission to reprint/republish this material for advertising or promotional purposes or for creating new collective works for resale or redistribution to servers or lists, or to reuse any copyrighted component of this work in other works must be obtained from the IEEE. 


\title{
Laser-Written Optical-Path Redirected Waveguide Device for Optical Back-Plane Interconnects
}

\author{
Seiki Hiramatsu, Member, IEEE, Takashi Mikawa, Osamu Ibaragi, Kiyotaka Miura, and Kazuyuki Hirao
}

\begin{abstract}
A novel optical-path redirected three-dimensional waveguide device for optical back-plane interconnects is demonstrated. To fabricate an optical waveguide device, a laser-written technique using focused irradiation of visible femtosecond pulses is employed. In this waveguide device, single-stroke drawing is applied for low-cost fabrication. To redirect the optical path, a planar reflective mirror was adopted. Since the focus of the laser was shifted successively while drawing cores in glass substrate, multichannel optical cores were continuously written up to the surface component. By having this structure, back-plane interconnects can be integrated more densely, and an optical back-plane board can be coupled to a daughter-board directly without any microlenses. Furthermore, all cores have the same length, so this device potentially suppresses the modulation skew caused by the difference of optical-path lengths. We present the fabrication and the test results of a glass-based waveguide device for optical-path redirection.
\end{abstract}

Index Terms-Back-plane interconnects, laser-written waveguide, matrix array, optical-path redirection, single-stroke writing.

\section{INTRODUCTION}

$\mathbf{I}_{\mathrm{r}}^{\mathrm{N}}$ $\mathrm{N}$ RECENT years, high bit-rate transmission has been required of local area networks and wide area networks. The wide variety of studies of coexisting technologies, e.g., wavelength-division multiplexing and time-division multiplexing, have greatly contributed to the field of long-distance communication [1], [2]. As a result of these efforts, the optical communication network of the Internet industry has experienced rapid growth. On the other hand, optical network devices such as servers and gigabits/s Ethernet modules needed to have multichannel inputs-outputs (I/Os) for higher integration and sufficient throughputs. Especially in optical interconnects using laser diodes and photodiodes for board-to-board and board-to-back-plane communications, optical devices with multichannel I/Os and cost-effective optical-path redirection have become the key technology for higher integration and low-cost fabrication. Although many devices for optical-path redirection using microlenses and spherical mirrors have been

Manuscript received December 29, 2003; revised May 16, 2004. This work was performed under the management of the Association of Super-Advanced Electronics Technologies (ASET) supported by the New Energy and Industrial Technology Development Organization (NEDO).

S. Hiramatsu, T. Mikawa, and O. Ibaragi are with Electronics System Integration Technology Research Development, Association of Super-Advanced Electronics Technologies, c/o NTT Musashino Research and Development Center, Tokyo 180-8585, Japan (e-mail: nasa@ diana.dti.ne.jp).

K. Miura is with Optical Device Development Department, Central Glass Co., Ltd., Yamaguchi 755-5199, Japan.

K. Hirao is with Division of Material Chemistry, Faculty of Engineering, Kyoto University, Kyoto 606-8501, Japan.

Digital Object Identifier 10.1109/LPT.2004.832587

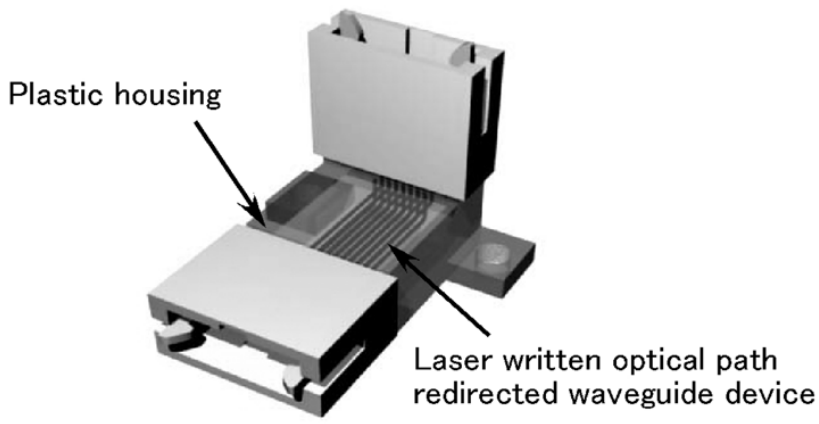

Fig. 1. Multichannel back-plane connector image having laser-written optical-path redirected waveguide device.

reported [3], [4], novel optical devices are required because the above components are costly elements in optical interconnects. One promising solution to this problem is to use a three-dimensional optical waveguide. Laser writing techniques using focused irradiation of visible femtosecond pulses that are nonresonant with absorption in glass substrate have been studied [5], [6]. By using this technique, three-dimensional optical waveguides that are difficult to fabricate by conventional dry etching can be written in glass substrate. A back-plane connector image of a laser-written optical-path redirected waveguide device in the plastic housing is shown in Fig. 1. This connector has matrix-arrayed multichannel optical I/Os and these optical paths are redirected by $90^{\circ}$. This optical-path redirected connector exploiting the three-dimensional waveguide can be coupled to a conventional optical connector. For example, a commercially available mechanically transferable connector having matrix-arrayed multichannel optical I/Os can be plugged to this connector easily [7]. Optical-path redirected three-dimensional waveguide devices having multichannel I/Os could integrate systems more densely and reduce costs of optical back-plane interconnects.

In this letter, we present the fabrication and test results of a laser-written optical-path redirected waveguide device having multichannel optical I/Os.

\section{FABRICATION}

\section{A. Laser-Written Waveguide}

To write optical waveguides in bulk glass, a femtosecond laser pulse was focused (shown in Fig. 2). Ultrashort pulses in the Gaussian mode produced by a regeneratively amplified $\mathrm{Ti}$ : sapphire laser that emits a $250-\mathrm{kHz}$ mode-locked pulse were utilized. The laser pulsewidth focused in the glass substrate was adjusted to 400 fs by changing the prism disperse path length in the compressor. The average laser pulse power at the sample 
(a)

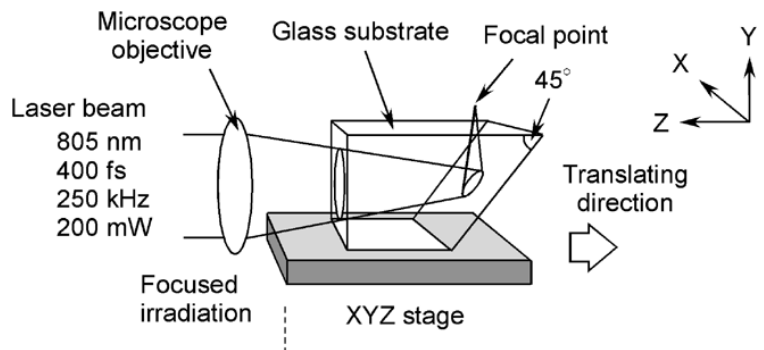

(b)

(c)

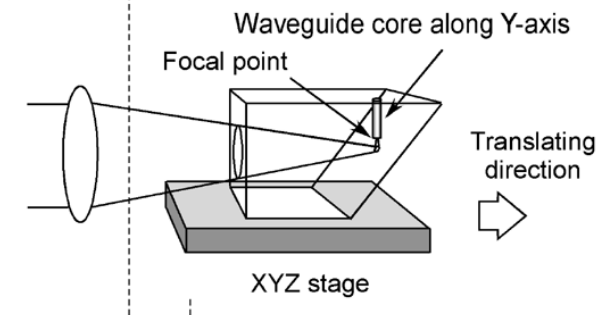

Waveguide core along $Y$-axis

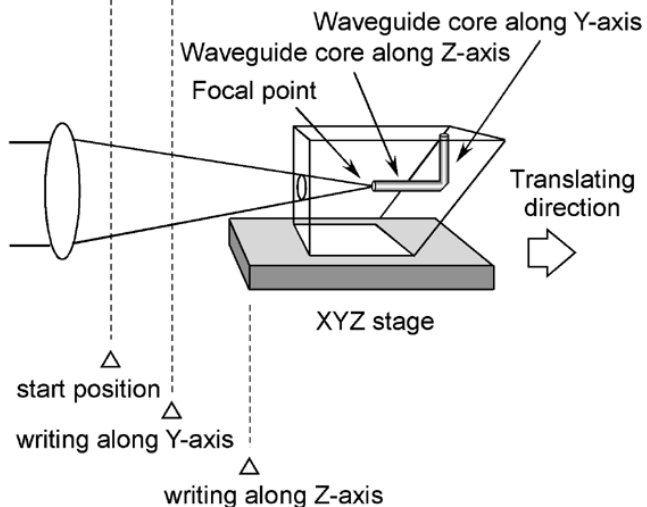

Fig. 2. Schematic illustration of leser writing process in the glass substrate (a) Start position of single strock writing. Focal point was addressed outside of the glass substrate. (b) Laser writing the waveguide core in $y$ axis. By translating the glass sample along the $z$ axis, the waveguide core was continuosly written and after reflection by mirror. (c) Laser writing the waveguide core along the $z$ axis.

location was approximately controlled to be $200 \mathrm{~mW}$. To write the optical waveguides, the laser beam was focused at a diameter of $5 \mu \mathrm{m}$ or less through a microscope objective and directed into the polished synthetic silica sample. An $x y z$ stage was controlled precisely with the help of computer. Single-mode waveguides were written by translating the sample at $50 \mu$ s along the axis of the laser beam.

\section{B. Optical-Path Redirected Device}

To fabricate an optical-path redirected waveguide device with low cost and low device loss, single-stroke writing was applied (shown in Fig. 2). A synthetic silica block having a $45^{\circ}$ edge was employed to redirect the optical path by $90^{\circ}$. In order to address the focal point outside of the synthetic silica block at the start position of a single-stroke writing, a focused femtosecond pulsed laser beam was redirected by a reflective mirror [shown in Fig. 2(a)]. By translating the glass block in the $z$ axis direction with sufficient care, the focal point was moved along the $y$ axis and the waveguide core along the $y$ axis direction could be written [shown in Fig. 2(b)]. After that, by translating the

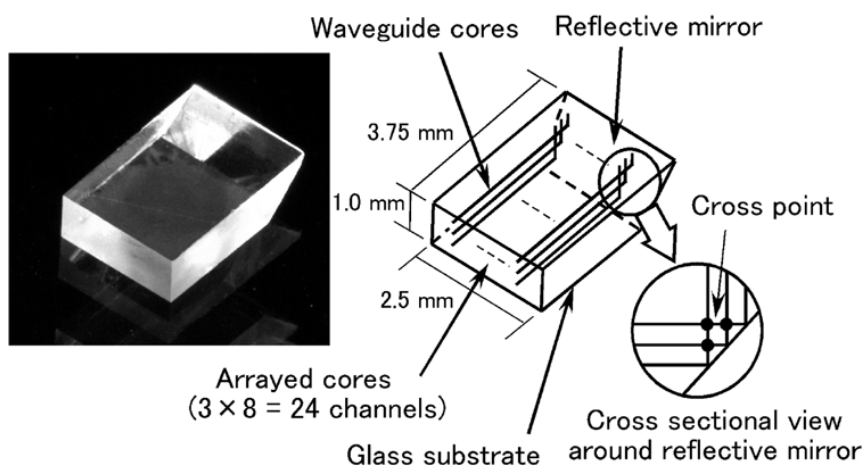

Fig. 3. Photograph and configuration of the laser-written optical-path redirected waveguide device

glass block further along the $z$ axis, the focal point was successively shifted before and after reflection by the mirror and the waveguide core was written continuously at the point of the optical-path redirection. A waveguide core in the $z$ axis direction was written to the surface of the component by translating the glass block further [shown in Fig. 2(c)]. Multichannel cores were written in the glass block by repeating the same process. This single-stroke writing reduces the fabrication costs in comparison with writing the waveguide core along the $y$ and $z$ axes, respectively, and potentially suppresses the optical loss because waveguide cores were continuously written before and after reflection by the mirror without fail.

\section{EXPERIMENTAL RESULTS}

In the fabricated waveguide device, 24-channel optical cores were written (shown in Fig. 3). The three cores along the $y$ axis and the eight cores along the $x$ axis were arrayed in this device. A waveguide core having optical-path redirection has two cross points derived from adjacent cores. By having such a structure, optical interconnects in the board-to-board and board-to-backplane communication structure can be integrated more densely. Furthermore, all cores in this device have the same length of $3.75 \mathrm{~mm}$, so this device potentially suppresses the modulation skew caused by the difference of optical-path length. 24-channel optical cores were arrayed with a $250-\mu \mathrm{m}$ pitch along the $x$ and $y$ axis for multichannel fiber coupling. The size of the device was $2.5 \times 3.75 \times 1.0 \mathrm{~mm}$.

We observed the near-field pattern (NFP) at $1310 \mathrm{~nm}$ for the waveguide device. The NFP was measured by a charged coupled device camera attached to a microscope with a $50 \times$ objective. After a single-mode fiber was coupled to one end of the core, the light was redirected by a reflective mirror. The light emitted from another end of the core was captured. The NFP of the fabricated waveguide device is shown in Fig. 4. These patterns provide a two-dimensional view of the mode structure introduced by the laser-written waveguide and show a good Gaussian fit. According to this pattern, it was found that a fundamental mode is formed with circular symmetry in the glass substrate and the mode field diameter (MFD) for the waveguide was approximately $8.3 \mu \mathrm{m}$. The far-field pattern was reduced by a $20 \times$ microscope objective and taken at a $2-\mathrm{mm}$ distance. This result indicates that the numerical aperture (NA) of the waveguide device was 0.098 . From the divergence of the waveguide 


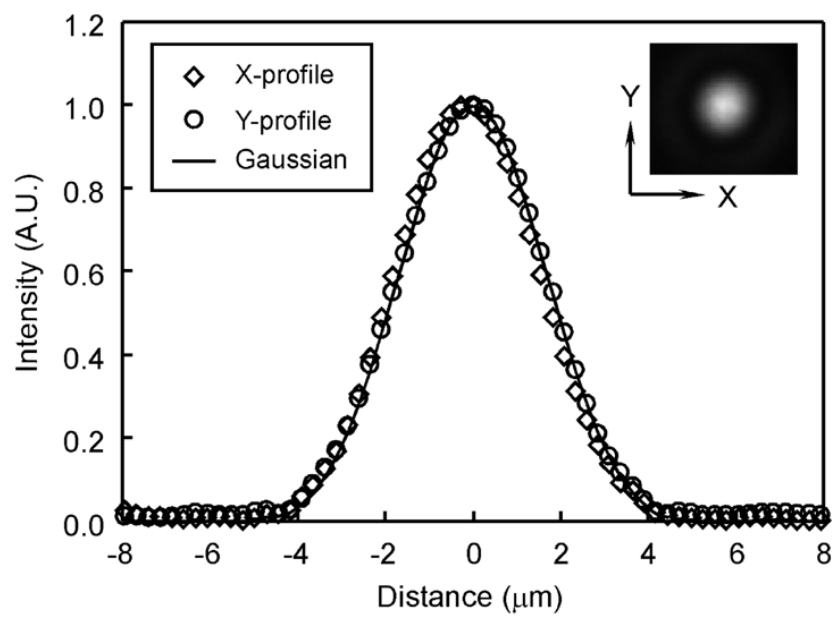

Fig. 4. NFP of the laser-written optical-path redirected waveguide device.

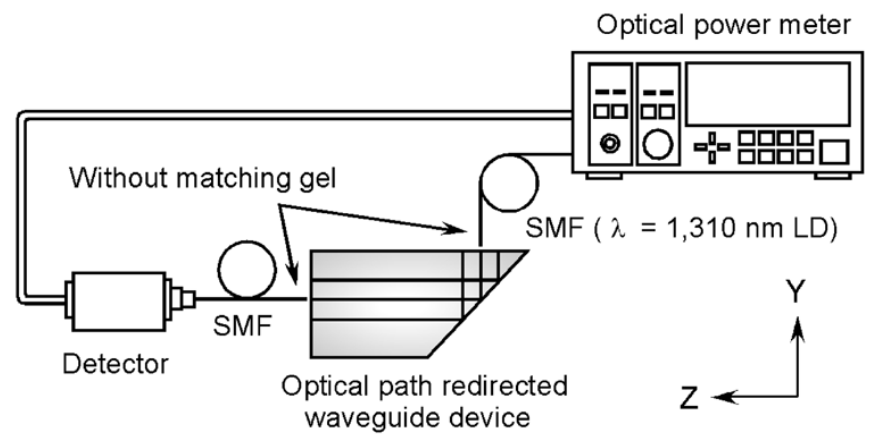

Fig. 5. Experimental setup for the optical loss measurement. In this measurement, matching gel was not employed

output, we found that the refractive index change between the core of the waveguide and the glass substrate was estimated to be approximately 0.003 .

We confirmed that the MFD and NA measured by a linearly written waveguide without optical-path redirection and cross points were almost the same as the fabricated device, so the optical signal induced from one end of the core could be redirected and propagated to the other end without changing the MFD and NA. This result indicates that waveguide cores can successfully connect to the optical fiber without microlenses for adjusting the beam pattern between the single-mode fiber and waveguide core, and there is little influence at the cross point of the core. The optical loss of the fabricated waveguide device was mea- sured by the butt-joint technique with the single-mode fiber at $1310 \mathrm{~nm}$ (shown in Fig. 5). In this measurement, matching gel was not employed.

The optical loss was as low as $1.1 \mathrm{~dB}$ on average. The measured propagation loss of the laser-written waveguide was about $0.9 \mathrm{~dB} / \mathrm{cm}$ and the optical loss derived from propagation in the fabricated waveguide device having a core length of $3.75 \mathrm{~mm}$ was estimated to be about $0.3 \mathrm{~dB}$. The optical loss except for the propagation loss in this device is presumably due to the reflective mirror loss for optical-path redirection and the coupling loss between the single-mode fiber and the waveguide device. The loss value scattering for each channel was about $\pm 10 \%$.

\section{CONCLUSION}

We have demonstrated for the first time a laser-written threedimensional waveguide device with an optical-path redirection by using single-stroke writing. This device has multichannel optical I/Os and the pitches were $250 \mu \mathrm{m}$ along the $x$ and $y$ axes. We confirmed the fabricated waveguide device provided adequate uniformity in the MFD and NA, and fairly low device loss for each channel. From the experimental measurements, it was found that these waveguide cores could connect optical fibers without microlenses. These results indicate that it is successfully functional to utilize the fabricated waveguide device as a cost-effective multichannel optical connector for optical back-plane interconnects.

\section{REFERENCES}

[1] H. Ishio, J. Minowa, and K. Nosu, "Review and status of wavelength-division-multiplexing technology and its application," J. Lightwave Technol., vol. LT-2, pp. 448-463, Apr. 1984.

[2] N. S. Bergano and C. R. Davidson, "Wavelength division multiplexing in long-haul transmission systems," J. Lightwave Technol., vol. 14, pp. 1349-1355, June 1996.

[3] J. Moisel, H.-P. Huber, J. Guttmann, O. Krumpholz, B. Lunitz, M. Rode, and R. Schoedlbauer, "Optical backplane," in Eur. Conf. Optical Communication, vol. 3, 2001, pp. 254-255.

[4] Y. Ishii, S. Koike, Y. Arai, and Y. Ando, "SMT-compatible optical-I/O chip packaging for chip-level optical interconnects," in Electronics Components and Technology Conf., 2001, pp. 231-237.

[5] K. M. Davis, K. Miura, N. Sugimoto, and K. Hirao, "Writing waveguides in glass with a femtosecond laser," Opt. Lett., vol. 21, no. 21, pp. 1729-1731, 1996.

[6] K. Miura, J. Qiu, T. Mitsuyu, and K. Hirao, "Preparation and optical properties of fluoride glass waveguides induced by laser pulses," J. NonCryst. Solids, vol. 256-257, pp. 212-219, 1999.

[7] M. Takaya and K. Shibata, "Design and performance of very-high-density 60-fiber connectors," J. Lightwave Technol., vol. 21, pp. 1549-1556, June 2003. 